\title{
Systematic analysis of the lysine acetylome reveals diverse functions of lysine acetylation in the oleaginous yeast Yarrowia lipolytica
}

\author{
Guangyuan Wang ${ }^{1 \dagger}$, Lizhong Guo ${ }^{1 \dagger}$, Wenxing Liang ${ }^{2}$, Zhenming $\mathrm{Chi}^{3}$ and Lin Liu ${ }^{1 *}$
}

\begin{abstract}
Lysine acetylation of proteins, a major post-translational modification, plays a critical regulatory role in almost every aspects in both eukaryotes and prokaryotes. Yarrowia lipolytica, an oleaginous yeast, is considered as a model for biooil production due to its ability to accumulate a large amount of lipids. However, the function of lysine acetylation in this organism is elusive. Here, we performed a global acetylproteome analysis of Y. lipolytica ACA-DC 50109. In total, 3163 lysine acetylation sites were identified in 1428 proteins, which account for $22.1 \%$ of the total proteins in the cell. Fifteen conserved acetylation motifs were detected. The acetylated proteins participate in a wide variety of biological processes. Notably, a total of 65 enzymes involved in lipid biosynthesis were found to be acetylated. The acetylation sites are distributed in almost every type of conserved domains in the multi-enzymatic complexes of fatty acid synthetases. The provided dataset probably illuminates the crucial role of reversible acetylation in oleaginous microorganisms, and serves as an important resource for exploring the physiological role of lysine acetylation in eukaryotes.
\end{abstract}

Keywords: Lysine acetylation, Acetylproteome, Yarrowia lipolytica, Oleaginous yeast, Lipid biosynthesis

\section{Introduction}

Post-translational modifications (PTMs) play an important role in modulating diverse cellular processes, which can change protein functions by introducing new functional groups such as phospho, acetyl, ubiquityl and methyl groups (Huang et al. 2015; Santos et al. 2011). Among them, acetylation of lysine is a highly dynamic and reversibly regulated PTM, which occurs on either the $\alpha$-amino group at the $\mathrm{N}$-terminus of the protein or the $\varepsilon$-amino group on the side chain of lysine residues (Choudhary et al. 2009; Wang et al. 2010; Mischerikow and Heck 2011). Lysine acetylation was first discovered in histone proteins (Allfrey et al. 1964; Phillips 1963) and its role has been extensively investigated in regulating

\footnotetext{
*Correspondence: liulin@qau.edu.cn

${ }^{\dagger}$ Guangyuan Wang and Lizhong Guo contributed equally to this work ${ }^{1}$ College of Life Sciences, Shandong Province Key Laboratory of Applied Mycology, Qingdao Agricultural University, Qingdao 266109, China Full list of author information is available at the end of the article
}

gene transcription by histone acetyltransferases (HATs) or histone deacetylases (HDACs) modifying the core histone tails (Lee and Workman 2007; Yang and Seto 2008). However, in addition to histones, many other proteins in nucleus, cytoplasm, mitochondria, and other cellular compartment, were also found to be acetylated (Spange et al. 2009), which were involved in regulating a wide variety of important cellular processes, such as enzymatic activity (Nambi et al. 2013; Starai and Escalante-Semerena 2004), protein interactions (Choudhary et al. 2009) and metabolic pathways (Wang et al. 2010).

In recent years, utilization of microbial oils produced by oleaginous yeasts as sources in large-scale operations presents a great industrial interest. These microbial oils have been considered as one of the most suitable substitute for biodiesel production. And biodiesel has been well documented to have many advantages over the conventional diesel because of its biodegradable, non-toxic, and essentially free of sulfur and aromatic components (Helwani et al. 2009). Although several oleaginous yeasts, 
including Cryptococcus curvatus, Lipomyces starkeyi, Rhodosporidium toruloides, Trichosporon capitatum and Yarrowia lipolytica, can accumulate lipids more than $20 \%$ of their dry cell biomass (Papanikolaou and Aggelis 2011), the regulatory mechanism of lipid biosynthesis in oleaginous yeasts is poorly studied. Advances in liquid chromatography-mass spectrometry (LC-MS/MS) and lysine-acetylated peptide immune precipitation enable lysine acetylation to be investigated on a proteomic scale (Mo et al. 2015). So far a large number of lysine-acetylated proteins have been proved to be involved in various cellular functions, especially in regulating central metabolic pathways. In Salmonella enterica, key enzymes controlling the direction of glycolysis versus gluconeogenesis and the branching between citrate cycle and glyoxylate bypass were all regulated by acetylation (Wang et al. 2010). In budding yeast Saccharomyces cerevisiae, 1059 acetylated proteins were identified, and a large proportion of acetylated proteins were implicated in the regulation of glycolysis/gluconeogenesis and amino acid metabolisms (Henriksen et al. 2012). We wondered whether lysine acetylation plays an important role in the regulation of the production of lipid biosynthesis in oleaginous yeast.

Yarrowia lipolytica ACA-DC 50109, an oleaginous yeast, could produce lipid almost $50 \%(\mathrm{w} / \mathrm{w})$ of the cell dry weight (Wang et al. 2013, 2015). In this study, we performed the first investigation on the acetylproteome of this microorganism. In total, we identified 3163 lysine acetylation sites in 1428 proteins, which account for $22.1 \%$ of the total proteins in the cell. The acetylated proteins are associated with diverse biological functions and cellular processes. Fifteen conserved acetylation motifs were detected through bioinformatics analysis of the sequences surrounding the acetylation sites. In addition, many enzymes involved in lipid biosynthesis were acetylated. These results provide a systems-wide view of acetylproteome and represent a dataset for functional analysis of acetylated proteins in Y. lipolytica.

\section{Materials and methods}

\section{Strains, media and culture}

The oleaginous yeast Y. lipolytica strain ACA-DC 50109 (Collection Number 2E00680 at the Marine Microorganisms Culture Collection of China), was kindly provided by Dr. Seraphim Papanikolaou (Papanikolaou and Aggelis 2003), was grown in yeast peptone dextrose (YPD) $(10.0 \mathrm{~g} / \mathrm{L}$ yeast extract, $20.0 \mathrm{~g} / \mathrm{L}$ bacto peptone, and $20.0 \mathrm{~g} / \mathrm{L}$ glucose). The compositions of the medium for lipid production were $60.0 \mathrm{~g} / \mathrm{L}$ glucose, $7.0 \mathrm{~g} / \mathrm{L} \mathrm{KH}_{2} \mathrm{PO}_{4}$, $2.5 \mathrm{~g} / \mathrm{L} \mathrm{Na}_{2} \mathrm{HPO}_{4}, 1.5 \mathrm{~g} / \mathrm{L} \mathrm{MgSO}{ }_{4} \cdot 7 \mathrm{H}_{2} \mathrm{O}, 0.15 \mathrm{~g} / \mathrm{L} \mathrm{CaCl}_{2}$, $0.15 \mathrm{~g} / \mathrm{L} \mathrm{FeCl}_{3} \cdot 6 \mathrm{H}_{2} \mathrm{O}, 0.02 \mathrm{~g} / \mathrm{L} \mathrm{ZnSO}_{4} \cdot 7 \mathrm{H}_{2} \mathrm{O}, 0.06 \mathrm{~g} / \mathrm{L}$ $\mathrm{MnSO}_{4} \cdot \mathrm{H}_{2} \mathrm{O}$, and $0.5 \mathrm{~g} / \mathrm{L}$ yeast extract (Wang et al. 2012).
The yeast strain was incubated and harvested according to the methods described (Wang et al. 2015). In brief, $2.0 \mathrm{~mL}$ of yeast cell suspension $\left(2.5 \times 10^{8}\right.$ cells $\left./ \mathrm{mL}\right)$ which had been pre-cultivated in YPD medium for $24 \mathrm{~h}$ were added to $50 \mathrm{~mL}$ of the lipid production medium. The culture was incubated on a rotary shaker at $180 \mathrm{rpm}$ and $28{ }^{\circ} \mathrm{C}$ for $72 \mathrm{~h}$. After that, cells in the culture broth were harvested by centrifugation $(8000 \times g$ for $5 \mathrm{~min})$ and washed three times with sterile distilled water.

\section{Lipid determination and observation of lipid particles in the yeast cells}

The total lipids in the cells $(1.0 \mathrm{~g})$ were extracted according to the methods described (Folch et al. 1957). The extracted lipids were weighted and oil content per $100 \mathrm{~g}$ of cell dry weight was calculated. The lipid particles in the yeast cells were stained by Nile Red and observed according to the methods described (Wang et al. 2012).

\section{Protein extraction and trypsin digestion}

After the yeast cells were grinded in liquid nitrogen, the protein in the sample was extracted according to the procedures described (Li et al. 2016; Liu et al. 2016). Protein concentration extracted above was determined using a 2-D Quant kit (GE Healthcare) according to the manufacturer's instructions. For digestion, the protein solution was reduced with dithiothreitol (DTT) $(5 \mathrm{mM})$ for $30 \mathrm{~min}$ at $56{ }^{\circ} \mathrm{C}$ and alkylated using iodoacetamide (IAA) $(20 \mathrm{mM})$ at room temperature for $45 \mathrm{~min}$ in darkness. Finally, the resulting sample was digested using trypsin (Li et al. 2016; Liu et al. 2016).

\section{Affinity enrichment}

Samples obtained as described in the previous section was separated into 6 fractions by high $\mathrm{pH}$ reversephase HPLC using an Agilent Systerm with a ZORBAX 300Extend-C18 column (5 $\mu \mathrm{m}$ particles, $4.6 \mathrm{~mm}$ ID, $250 \mathrm{~mm}$ length). Acetylated peptides in each fraction were enriched using anti acetyllysine antibody beads (PTM Biolabs, Cat. No. 104, Hangzhou, China). Finally, the resulting peptides were cleaned with $\mathrm{C} 18$ ZipTips (Millipore) followed by LC-MS/MS analysis.

\section{LC-MS/MS analysis}

The acetylated peptides obtained above were dissolved in $0.1 \%$ formic acid (FA) and separated on a reversedphase Acclaim Pep-Map RSLC C18 column $(2 \mu \mathrm{m}$, $50 \mu \mathrm{m} \times 15 \mathrm{~mm}, 100 \AA$ ) (Thermo Scientific) by a gradient elution of $0.1 \%$ FA and $98 \%$ acetonitrile, with a flow rate of of $300 \mathrm{~nL} / \mathrm{min}$ on an EASY-nLC 1000 UPLC system (Zhou et al. 2016). The resulting peptides were subjected to electrospray/nanospray ionization (ESI/NSI) and analyzed by MS/MS using Q Exactive ${ }^{\mathrm{TM}}$ Plus tandem 
mass spectrometry (Thermo Scientific). The electrospray voltage applied was $2.0 \mathrm{kV}$. The $\mathrm{m} / \mathrm{z}$ scan range was 350-1800 for full scan, and intact peptides were detected in the Orbitrap at a resolution of 70,000. Peptides were then selected for MS/MS using NCE setting as 30 and the fragments were detected in the Orbitrap at a resolution of 17,500. A data-dependent procedure that alternated between one MS scan followed by $20 \mathrm{MS} / \mathrm{MS}$ scans with $15.0 \mathrm{~s}$ dynamic exclusion. Automatic gain control (AGC) was set at $5 \mathrm{E} 4$.

\section{Database search}

MaxQuant with integrated Andromeda search engine (v.1.4.2) was employed to process the resulting MS/MS data (Cox and Mann 2008; Cox et al. 2009). Tandem mass spectra were searched against UniProt-Y. lipolytica database (6453 sequences) concatenated with reverse decoy database. Trypsin/P was specified as cleavage enzyme allowing up to 4 missing cleavages. The mass tolerance for precursor ions was set as $20 \mathrm{ppm}$ in First search and $5 \mathrm{ppm}$ in Main search, and the mass tolerance for fragment ions was set as $0.02 \mathrm{Da}$. Carbamidomethyl on Cys was specified as fixed modification and acetylation on Lys and oxidation on Met were specified as variable modifications. False discovery rate (FDR) was adjusted to $<1 \%$ and the site localization probability was set as $>0.75$.

\section{Bioinformatics analyses}

For functional annotations of all the acetylated proteins obtained in the previous section, enrichment analyses based on Gene Ontology (GO) (http://www.ebi.ac.uk/ GOA) and Kyoto Encyclopedia of Genes and Genomes (KEGG) pathways were investigated (Kanehisa et al. 2004), respectively. Functional description of protein domains was annotated by InterProScan based on protein sequence alignment method, and the InterPro domain database was used (Hunter et al. 2009). Wolfpsort, a subcellular localization predication soft, was used to predict subcellular localization of the identified acetylated proteins (Horton et al. 2007). DAVID bioinformatics resources 6.7 was used to identify GO term, KEGG pathway and protein domain (Jiao et al. 2012). A two-tailed Fisher's exact test was used to test specific annotation terms among members of resultant protein clusters. Any annotation categories with Fisher's exact test $p$ value $<0.05$ were considered strongly enriched. Motif-x software was employed to analyze the model of sequences constituted with amino acids in specific positions of acetyl-21-mers (10 amino acids upstream and downstream of the site) in all protein sequences (Chou and Schwartz 2011). Protein-protein interactions for the identified acetylated proteins were performed using Cytoscape software and the protein-protein interaction network was obtained from the STRING database according to the methods described previously (Shannon et al. 2003).

\section{SDS-PAGE and Western blotting}

The proteins extracted above were determined by sodium dodecyl sulfate polyacrylamide gel electrophoresis (SDSPAGE) and Western blotting. Briefly, $12 \%$ gel in SDSPAGE was used to separate the proteins with different molecular weight and then all the proteins were electrotransferred onto a polyvinylidene difluoride (PVDF) membrane using a Bio-Rad Trans-Blot system. $5 \%$ skim milk powder was used to block the membranes in TBST buffer $(20 \mathrm{mM}$ Tris- $\mathrm{HCl}, 150 \mathrm{mM} \mathrm{NaCl}, 0.05 \%$ Tween 20) for 30-60 min at room temperature. After that, the PVDF membranes were incubated with pan anti-acetyl lysine antibody (PTM Biolabs, 101), anti-histone H2A.Z antibody (Abcam, ab4174) and anti-H2A.Z (acetyl K7) antibody (Abcam, ab214730) (1:1000, in TBS/5\% skim milk powder) for $1 \mathrm{~h}$ at room temperature, respectively. After the PVDF membranes were washed 3 times with TBST buffer, the membranes were incubated with horseradish peroxidase (HRP) conjugated anti-rabbit IgG (1:5000 dilutions) for $1 \mathrm{~h}$ at room temperature. Last, an enhanced chemiluminescence (ECL) immunoblotting detection kit (Beyotime Biotechnology, Shanghai) was used for signal detection.

\section{Results}

Proteome-wide analysis of lysine acetylation sites and proteins in Y. lipolytica

Yarrowia lipolytica ACA-DC 50109 was cultured in the oil production medium. As shown in Fig. 1, $10.8 \mathrm{~g} / \mathrm{L}$ of the dry cell mass (Fig. 1a), 41.6\% (w/w) of lipid based on cell dry weight were obtained (Fig. 1b), respectively. And most of the cells contained big lipid bodies and were stained by Nile Red (Fig. 1c, d), indicating that ACA-DC 50109 could synthesize high-level lipid in the cells. To elucidate the regulatory functions of lysine acetylation in this oleaginous yeast, we performed a proteome-scale analysis of acetylated proteins and acetylated sites in $Y$. lipolytica. Overview of experimental procedures used in this study was shown in Fig. 2a. Proteins were extracted and digested with trypsin, and then total peptides were subjected to acetylated enrichment with anti-acetyllysine antibody beads and analyzed using high-resolution LC-MS/MS. To validate the MS data, the mass error of all the identified peptides were checked and the results showed that the distribution of mass error was near zero and most of them were less than $5 \mathrm{ppm}$ (Fig. 2b), which means the mass accuracy of the MS data fits the requirement. The length of most peptides was distributed between 7 and 25 , which agrees with the property of 

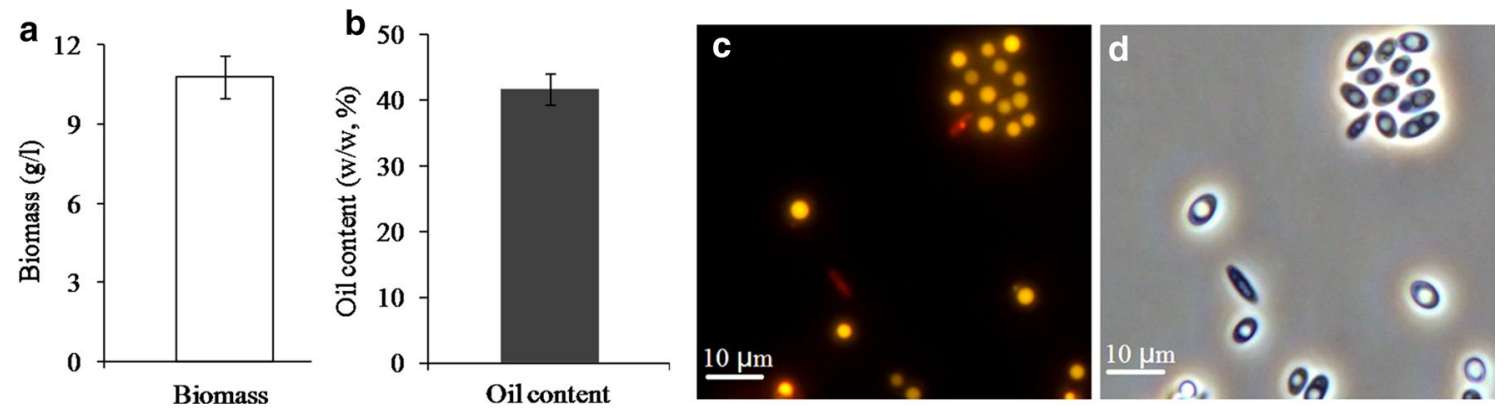

Fig. 1 Cell mass, oil content and lipid particles of the yeast strain ACA-DC 50109. a Biomass of the yeast strain. Data are given as mean \pm SD, $n=3$. b Oil content of the yeast strain. Data are given as mean $\pm S D, n=3$. c Lipid particles of the yeast strain were taken under fluorescent microscope. d Lipid particles of the yeast strain were taken under phase microscope

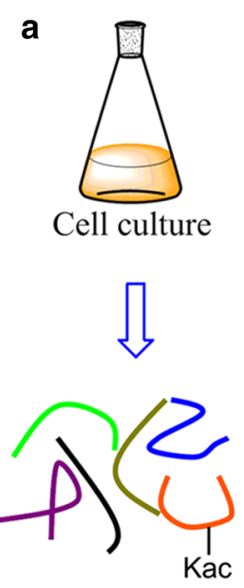

Lysis \& digestion<smiles>C1=CC=C1</smiles><smiles>CCCC(CC)CC</smiles>

Enrichment

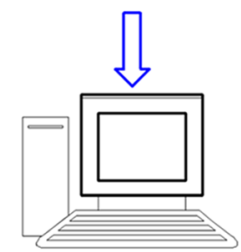

LC-MS/MS Analysis
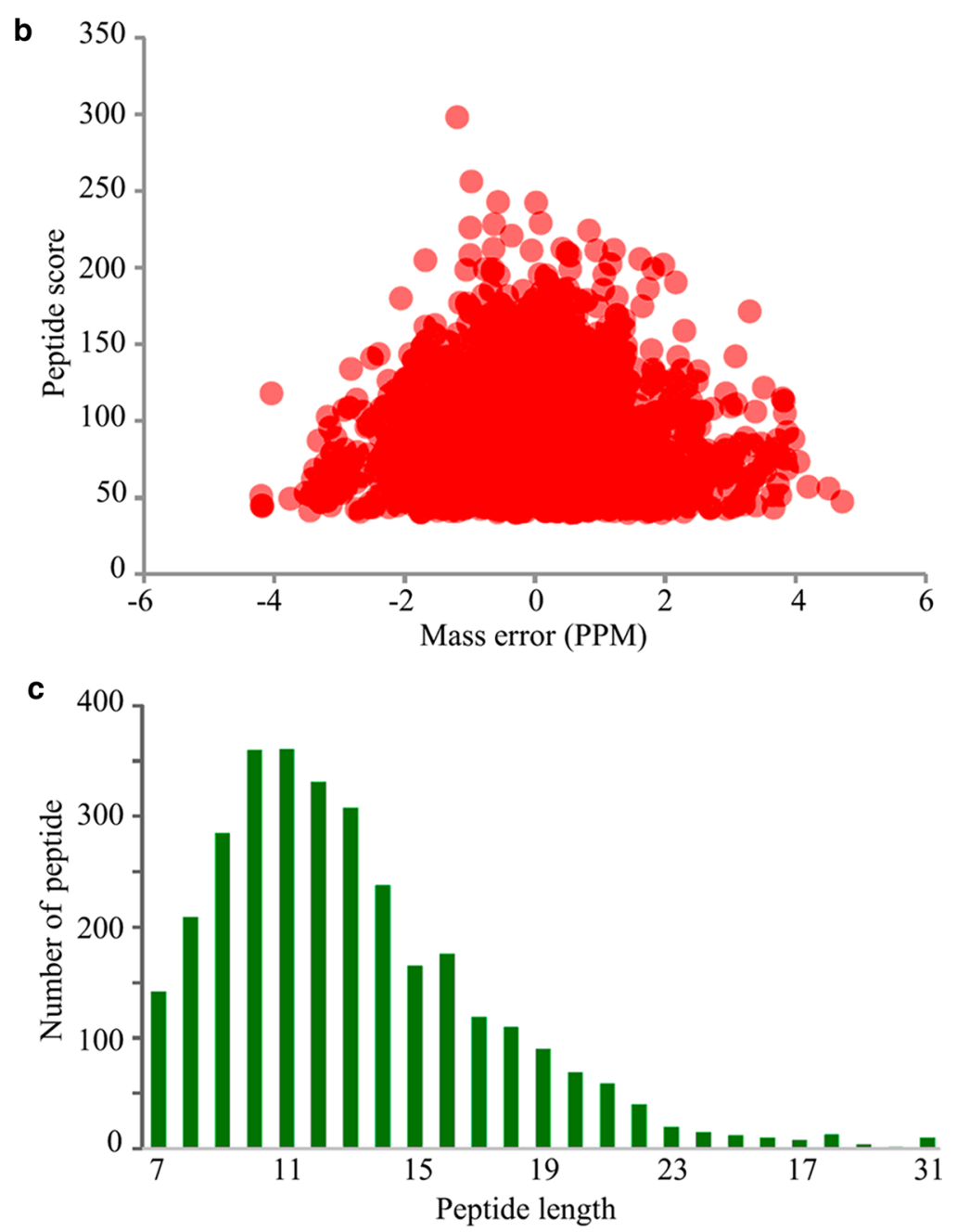

Fig. 2 Proteome-wide identification of lysine acetylation sites in Y. lipopytica. a Overview of experimental procedures used in this study. b Mass error distribution of all identified peptides. c Peptide length distribution 
tryptic peptides (Fig. 2c), and means that sample preparation reaches the standard. Additional file 1: Figure S1 shows three examples of MS/MS spectra of acetylated peptides. As such, totally 3163 lysine acetylation sites that matched to 1428 unique proteins from the UniProt_Y. lipolytica database were identified (Additional file 2: Table S1). The mass spectrometry proteomics data have been deposited to the ProteomeXchange Consortium via the PRIDE partner repository with the dataset identifier PXD006272.

To validate the diversity of acetylated proteins in $Y$. lipolytica, we performed Western blotting analysis using pan anti-lysine acetylation antibodies. As shown in Fig. 3a line 2, multiple immunoblot signals with a wide range of molecular weights were observed, indicating that acetylated proteins was abundant in $Y$. lipolytica. According to the LC-MS/MS analysis, an acetylated site at K7 of histone H2A.Z (Q6C341) was obtained (Additional file 2: Table S1; Fig. 3c). In order to confirm this acetylated site, we further performed Western blotting analysis using anti-histone H2A.Z (acetyl K7) antibody. Consistent with the MS/MS spectra of histone H2A.Z
(K7) (Fig. 3c), a legible band was observed after ECL immunoblotting detection (Fig. 3b), indicating the mass spectrometry proteomics data are reliable.

\section{Distribution and motif analysis of lysine acetylation sites}

In order to evaluate the distribution of acetylation sites in the acetylated proteins of $Y$. lipolytica, we calculated the numbers of identified modification sites per protein. The results showed that $52 \%$ of proteins contained only one acetylation site, and the percentage of proteins with two, three, four and five or more modification sites were 21 , 12,6 , and $9 \%$, respectively (Fig. $4 \mathrm{a}$ ). The heat map of different types of amino acids from -10 to +10 surrounding the acetylation sites was investigated. The frequency of phenylalanine (F), isoleucine (I), leucine (L) and tyrosine (Y) in positions -2 to +2 was highest, while the occurrence of lysine (K), glutamic acid $(\mathrm{E})$ and arginine $(\mathrm{R})$ was lowest (Fig. 4b).

To further assess the character of acetylated proteins in $Y$. lipolytica, the sequence motifs in all of the identified acetylated peptides were analyzed using the motif- $x$ program. Totally, 15 conserved sequences were identified

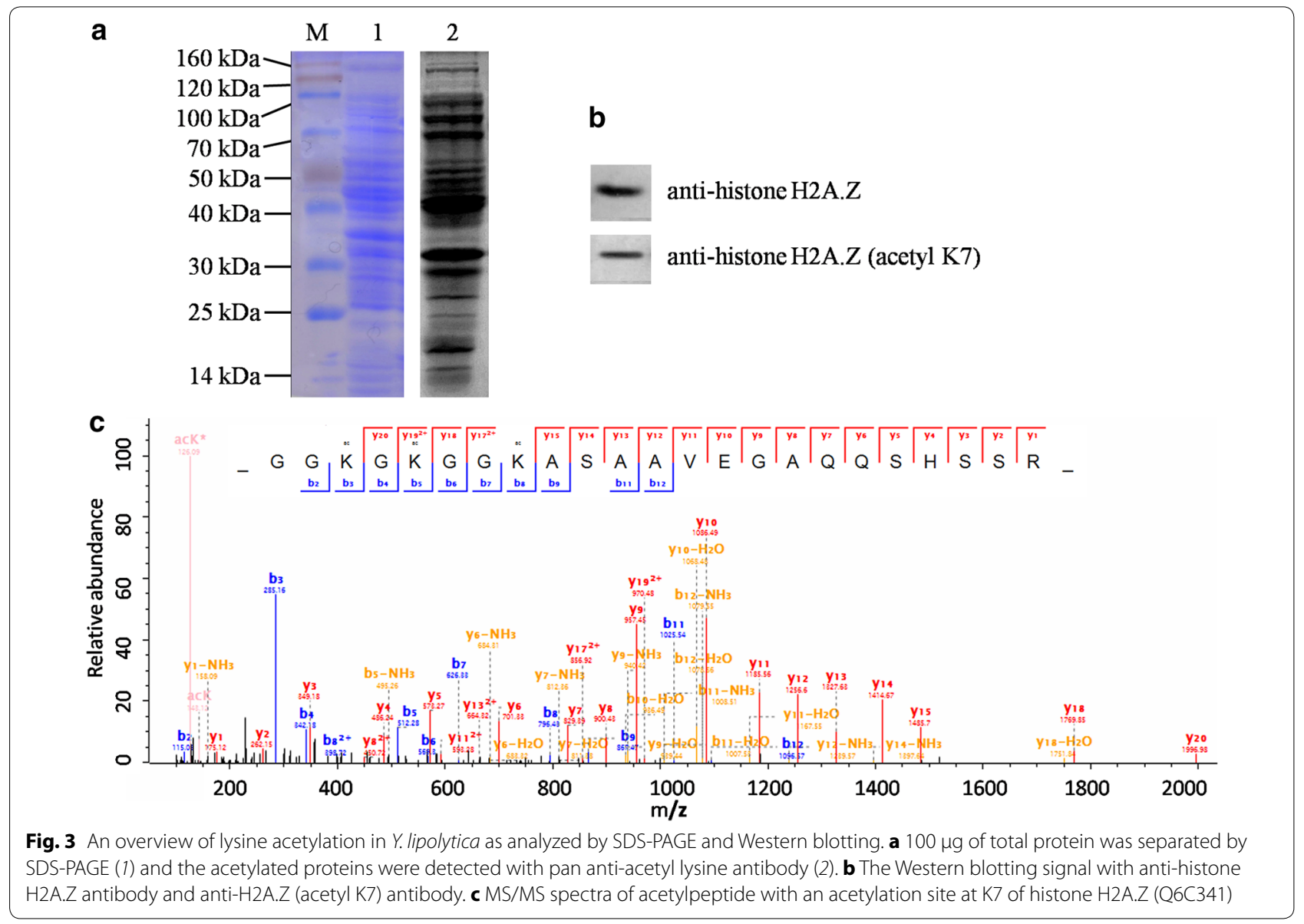




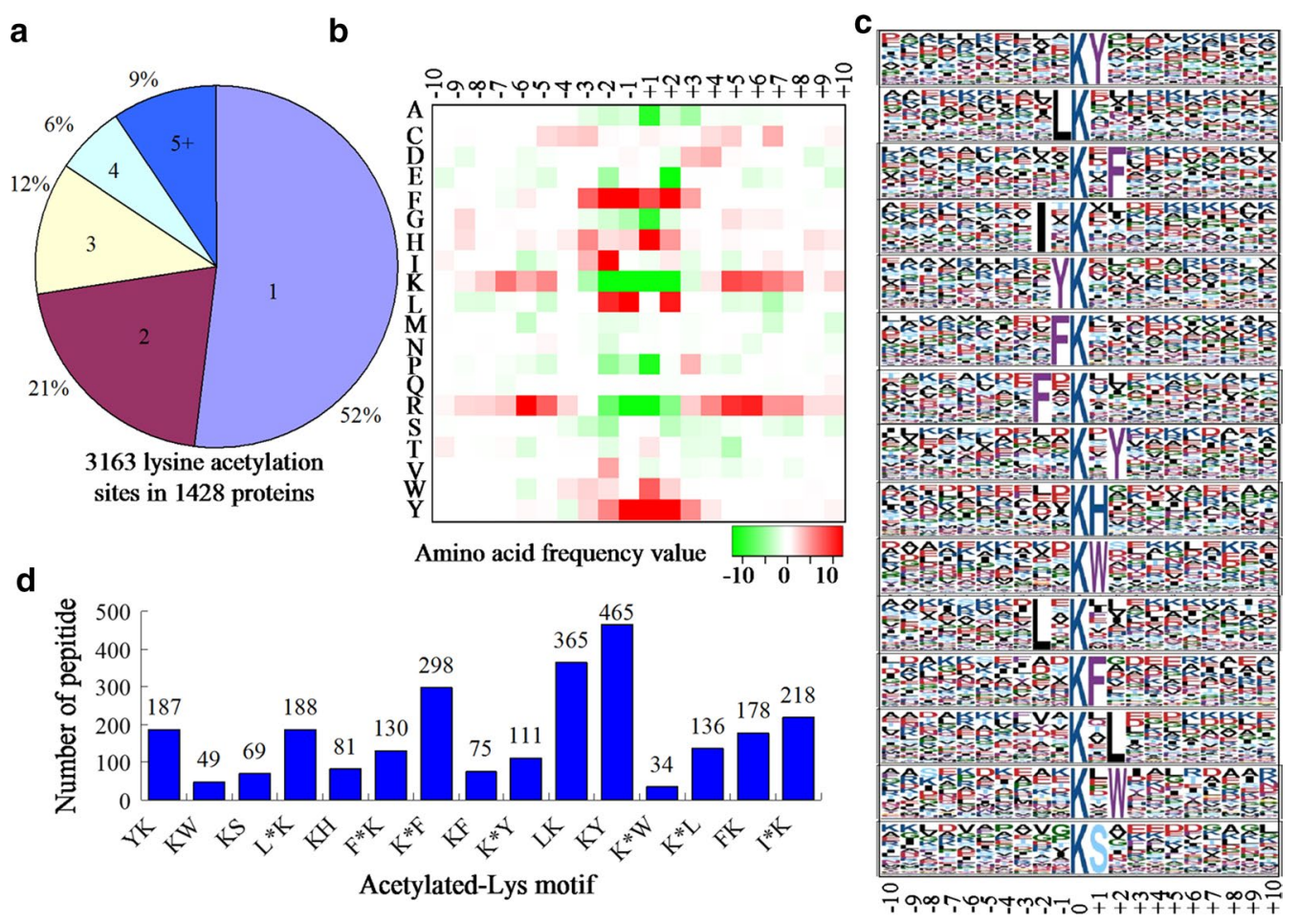

Fig. 4 Distribution and motif analysis of lysine acetylation sites. a Pie chart illustrating the number and percentage of lysine acetylation sites per protein. $\mathbf{b}$ Heat map of the amino acid compositions around the lysine acetylation sites showing the frequency of different types of amino acids surrounding this residue. $\mathbf{c}$ Sequence probability logos of significantly enriched acetylation site motifs for \pm 10 amino acids around the lysine acetylation sites. $\mathbf{d}$ Number of identified peptides contained in each conserved motif

(Fig. 4c), which exhibited different abundances (Fig. 4d). Importantly, although most of the motifs have been found in other microorganisms (Huang et al. 2015; Li et al. 2016; Liao et al. 2014; Liu et al. 2016; Lv et al. 2016; Pan et al. 2014; Xie et al. 2015), the significantly conserved motif, $\mathrm{K}^{\mathrm{ac}} \mathrm{XW}\left(\mathrm{K}^{\mathrm{ac}}\right.$ represents acetylated lysine and $\mathrm{X}$ represents a random amino acid residue) in Y. lipolytica, were identified for the first time. A survey of these motifs indicated that four distinct types of residues were found around the acetylated lysine: a residue with hydrophobic side chain groups, such as L and I; a residue with aromatic groups, including $\mathrm{Y}, \mathrm{F}$ and tryptophan (W); a positively charged residue, histidine $(\mathrm{H})$; and a polar amino acid residue, serine (S) (Fig. 4c).

\section{Secondary structure analysis of acetylated proteins}

To elucidate the relationship between the acetylated sites and protein structure, we performed a structural analysis of all the acetylated proteins in Y. lipolytica (Fig. 5). As shown in Fig. 5a, 32.6\% (1031) of the acetylation sites was located at regions with ordered secondary structures. Among them, 25.5\% (806) was located in $\alpha$-helix and 7.1\% (225) in beta-strand (Fig. 5a). In addition, 67.3\% (2132) of the acetylation sites was distributed in the disordered structures of proteins (Fig. 5a). Although the distribution pattern of all lysine was similar to that of acetylated lysine, the difference between them was significant $(p<0.001)$ (Fig. 5a), suggesting an interesting relationship between lysine acetylation and secondary structures. Surface accessibility of the acetylated lysine sites was also analyzed. The results in Fig. 5b showed that, compared with $37.92 \%$ of all lysine residues, $34.59 \%$ of the modified sites $(p=4.44 \mathrm{e}-16)$ was located on the protein surface. Thus, surface property of proteins is likely be changed by lysine acetylation in the oleaginous yeast $Y$. lipolytica.

\section{Functional annotation and cellular localization of acetylated proteins}

To better understand the lysine acetylome of Y. lipolytica, the GO functional classification of all the acetylated proteins based on their biological process, molecular function and cellular component was investigated (Fig. 6a-c). The GO analysis indicated that the acetylated proteins were distributed broadly in terms of their biological processes. Among them, the largest group is proteins associated with metabolic processes (36\%) and cellular process (36\%) (Fig. 6a). According to the GO molecular function category, the largest proportion of identified proteins 

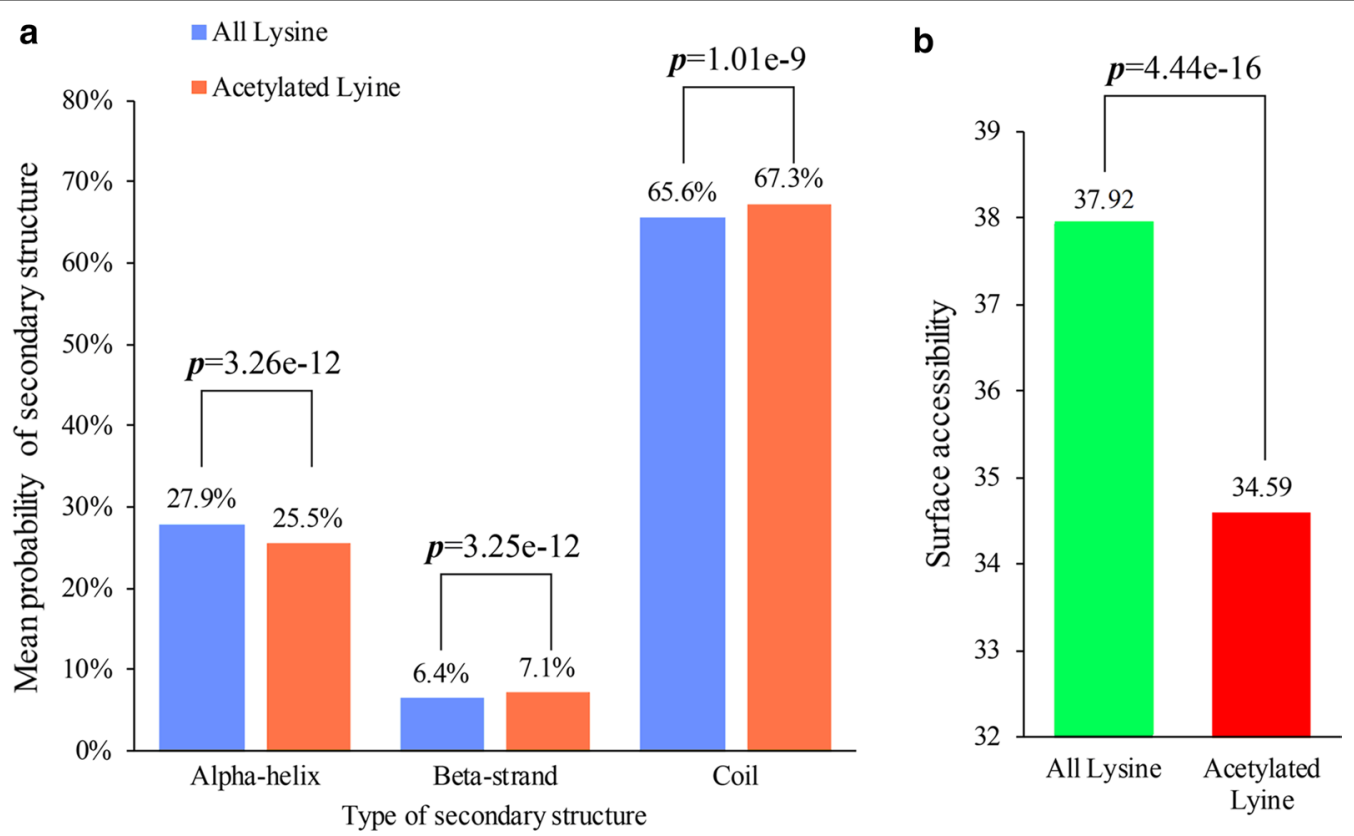

Fig. 5 Secondary structure analysis of acetylated proteins. a Probabilities of lysine acetylation in the structures of alpha-helix, beta-strand and coil. b Predicted surface accessibility of acetylation sites

were associated with catalytic activity (46\%) (Fig. 6b), and the second largest group of acetylated proteins was found to be related to binding (39\%) (Fig. 6b). The results of cellular component analysis showed that the acetylated proteins identified were distributed in organelles (45\%), macromolecular complex (28\%), membrane (21\%) and membrane-enclosed lumen (6\%) (Fig. 6c). As shown in Fig. 6d, most of the acetylated proteins in Y. lipolytica were distributed in the cytosol (35\%), mitochondria (26\%) and nucleus (23\%), as determined by subcellular localization analysis. Taken together, these data demonstrates that the lysine acetylated proteins in Y. lipolytica, which are located to various cellular components, participate in multiple functional processes.

\section{Functional enrichment analysis of acetylated proteins}

In order to further study what types of proteins are preferred targets for lysine acetylation, we performed a series of enrichment analyses based on GO, KEGG pathway and domain, respectively. According to $\mathrm{GO}$ functional enrichment, proteins with the molecular functions of structural molecule activity, structural constituent of ribosome, oxidoreductase activity, ligase activity, and nucleotide binding were found to be significantly enriched (Fig. 7a; Additional file 3: Table S2), which were associated with energy and substance metabolism. Consistent with these observations, proteins involved in organic acid metabolic process, carboxylic acid metabolic process, organic substance biosynthetic process, organonitrogen compound metabolic process, and translation were more likely to be acetylated based on the biological process analysis (Fig. 7a; Additional file 4: Table S3). The enrichment analysis based on cellular component showed that proteins distributed in cytoplasm, ribosome, macromolecular complex and non-membrane-bounded organelle have high tendency to be acetylated (Fig. 7a; Additional file 5: Table S4). In agreement with the results presented above, KEGG enrichment analysis indicated that proteins involved in citrate cycle, 2-oxocarboxylic acid metabolism, pyruvate metabolism, carbon metabolism and metabolic pathways were highly enriched (Fig. 7b; Additional file 6: Table S5). Moreover, domain enrichment analysis demonstrated that a large amount of acetylated proteins containing diverse domains, such as $\mathrm{NAD}(\mathrm{P})$ binding domain, ATP-grasp fold subdomain, pyridoxal phosphate-dependent transferase, aminoacyl-tRNA synthetase, thioredoxin-like fold, were found to be enriched (Fig. 7c; Additional file 7: Table S6). It was worthwhile to mention that ATP, NADPH and organic acid were essential materials for fatty acid biosynthesis. These findings suggest that multiple pathways, including intracellular fatty acid metabolism, may be strictly regulated by lysine acetylation in oleaginous yeast.

\section{Acetylated proteins related to lipid metabolism}

Functional enrichment analysis of identified acetylated proteins revealed that intracellular lipid metabolism may be regulated by lysine acetylation in Y. lipolytica. To 


\section{a}

localization $6 \%$ biological regulation $5 \%$

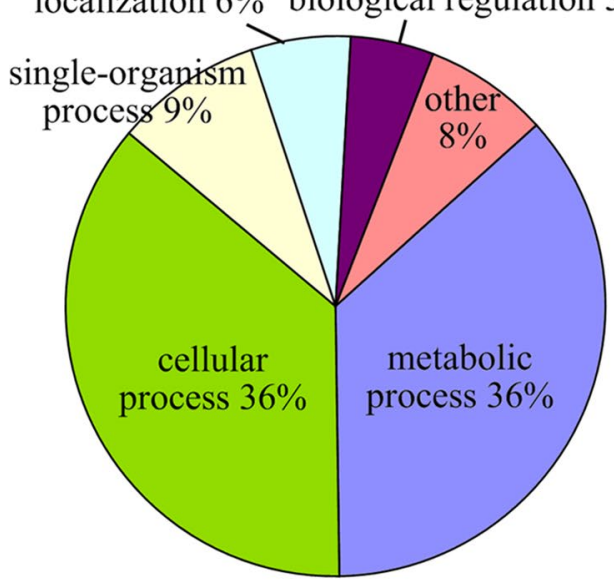

C

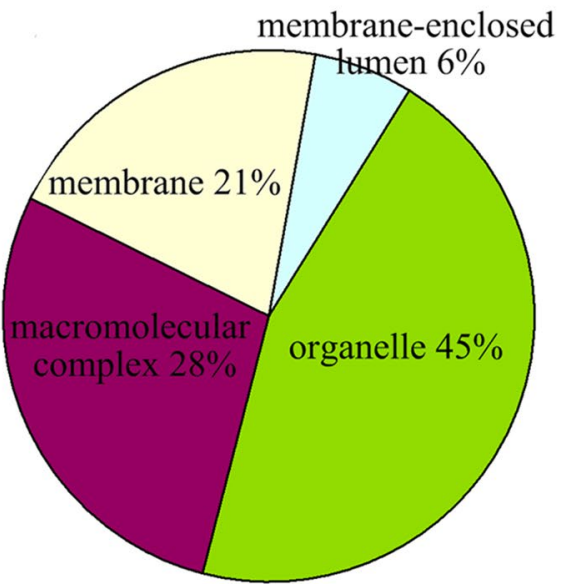

b electron carrier activity $2 \%$

transporter activity $3 \%$ /

structural molecule

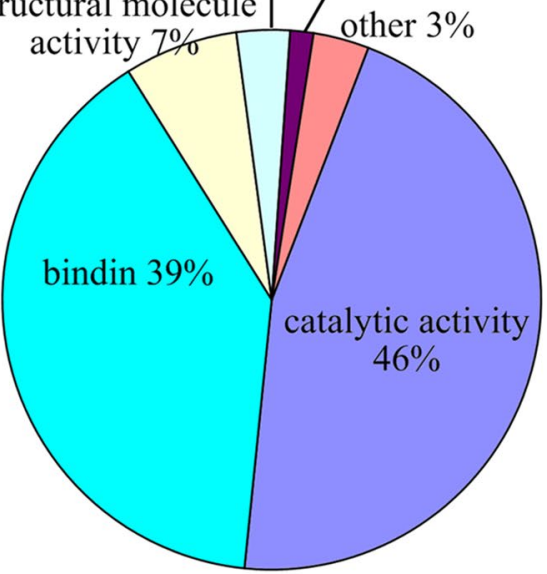

d $\quad$ other $2 \%$ plasma membrane $4 \%$

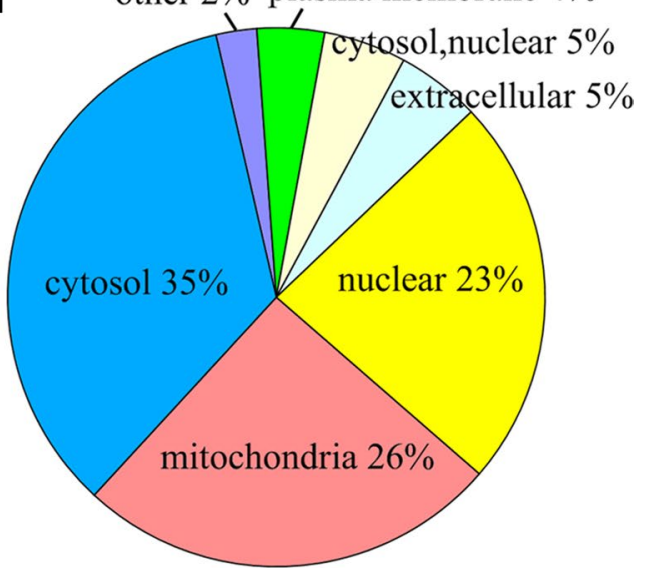

Fig. 6 Pie charts showing the functional classification of acetylated proteins in Y. lipolytica. a Classification of the acetylated proteins based on biological process. b Classification of the acetylated proteins based on molecular function. c Classification of the acetylated proteins based on cellular component. d Subcellular localization of the acetylated proteins

confirm these observations, we further analyzed the acetylated proteins involved in TAGs biosynthesis. Notably, a total of 65 enzymes involved in lipid biosynthesis were found to be acetylated (Fig. 8a; Additional file 8: Table S7). Our results that nearly all enzymes in major metabolic pathways, such as glycolysis, PPP, tricarboxylic acid (TCA) cycle, glyoxylate pathway, fatty acid biosynthesis and beta-oxydation are subjected to acetylation support the notion that acetylation may be linked to cellular metabolism at multiple levels.

Generally, the fatty acid chain grows through the addition of units of malonyl-CoA or acetyl-CoA, which is catalyzed by fatty acid synthetase (FAS), a multi-enzymatic complexes composed of two subunits, FAS1 (beta subunit) and FAS2 (alpha subunit) in Y. lipolytica (Wang et al. 2013). Interestingly, both FAS1 and FAS2 contain
12 acetylation sites in Y. lipolytica (Fig. 8b). Further study revealed that among these sites in FAS1, one (K219) is located in the domain of malonyl CoA-acyl carrier protein transacylase; three (K876, K1229 and K1285) are located in enoyl reductase domain of yeast-type FAS1; one (K1607) is located in the domain of MaoC; five (K1747, K1787, K2004 and K2051) are located in acyl transferase domain (Fig. 8b). In FAS2, one site (K64) in FabD domain, six sites (K542, K543, K687, K952, K1058 and K1085) in 3-oxoacyl-(acyl-carrier-protein) reductase domain, two sites (K1411, K1543) in FabB domain, and two sites (K1781, K1786) in AcpS domain, were identified (Fig. 8c). Collectively, the acetylation sites were found to be distributed in almost every types of conserved domains in the multi-enzymatic complexes of fatty acid synthetases. 
a

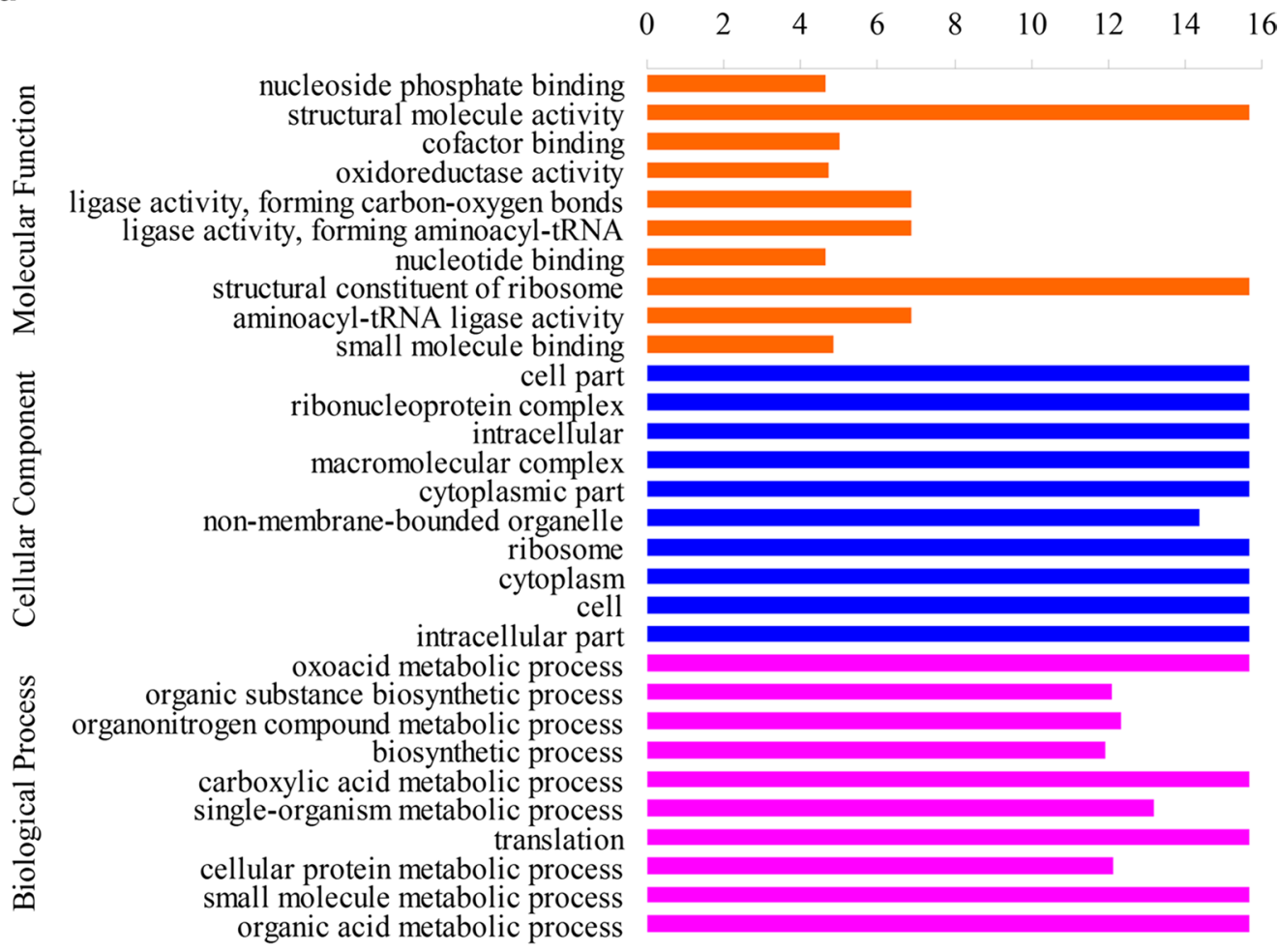

- $\log 10$ (Fisher's test $\mathrm{p}$ value)

b

Pyruvate metabolism

Biosynthesis of antibiotics

Biosynthesis of secondary

Biosynthesis of amino acids

Metabolic pathways

Carbon metabolism

Citrate cycle (TCA cycle) Ribosome

Aminoacyl-tRNA biosynthesis 2-Oxocarboxylic acid metabolism

$\begin{array}{lllllllll}0 & 2 & 4 & 6 & 8 & 10 & 12 & 14 & 16\end{array}$

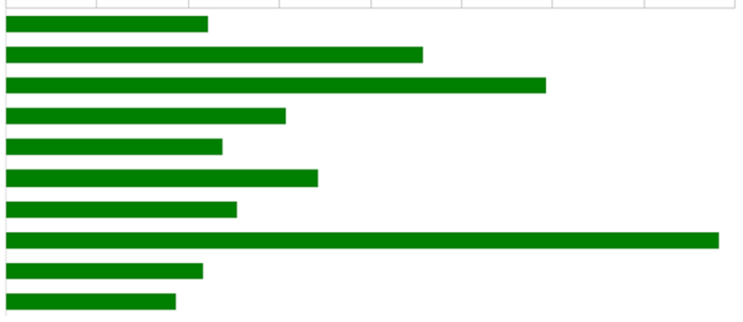

$-\log 10$ (Fisher's test $\mathrm{p}$ value)

C

NAD(P)-binding domain Thioredoxin-like fold

Heat shock protein $70 \mathrm{kD}$, C-terminal domain Pyridoxal phosphate-dependent transferase Aminoacyl-tRNA synthetase, class II Heat shock protein 70 , conserved site ATP-grasp fold, subdomain 2 Heat shock protein 70 family

Pyridoxal phosphate-dependent transferase Heat shock protein $70 \mathrm{kD}$, peptide-binding domain $\begin{array}{llllllll}0 & 1 & 2 & 3 & 4 & 5 & 6 & 7\end{array}$

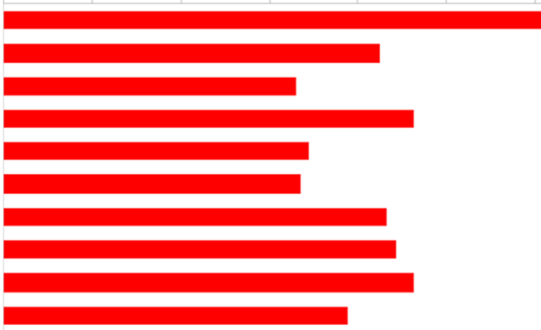

Fig. 7 Enrichment analysis of the acetylated proteins in Y. lipolytica. a GO-based enrichment analysis in terms of molecular function, cell component and biological process. b KEGG pathway-based enrichment analysis of the acetylated proteins. c Domain-based enrichment analysis of the acetylated proteins 
a

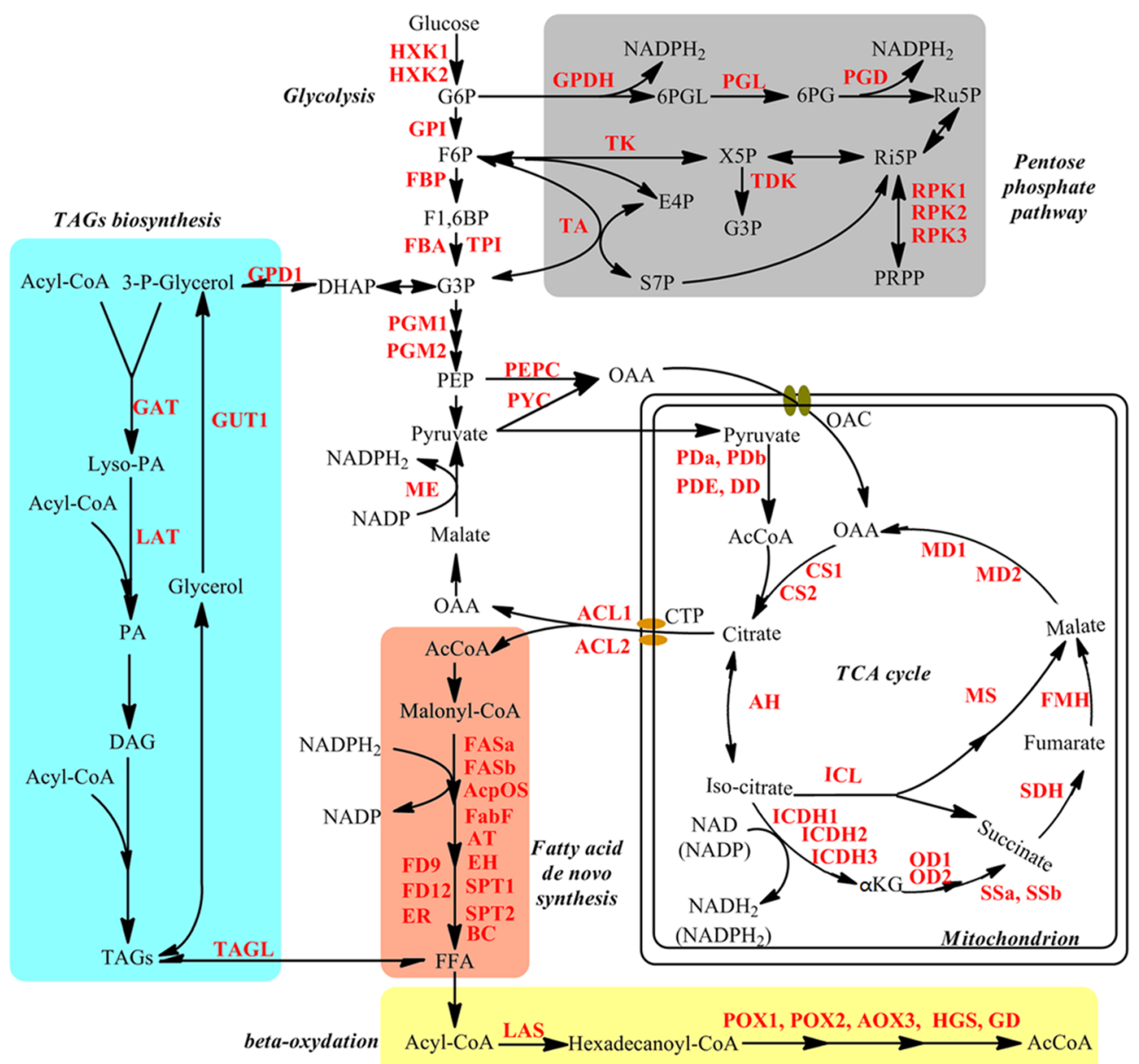

b

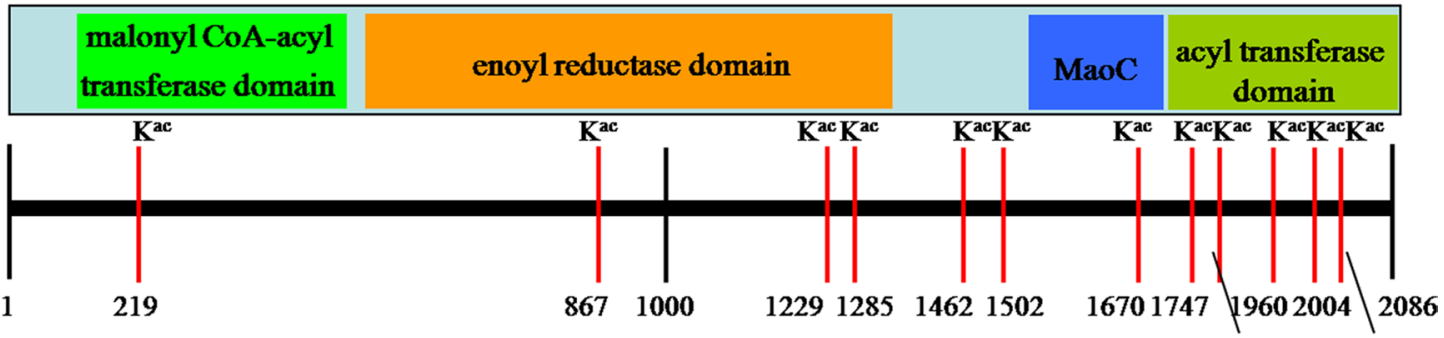

$1787 \quad 2051$

C

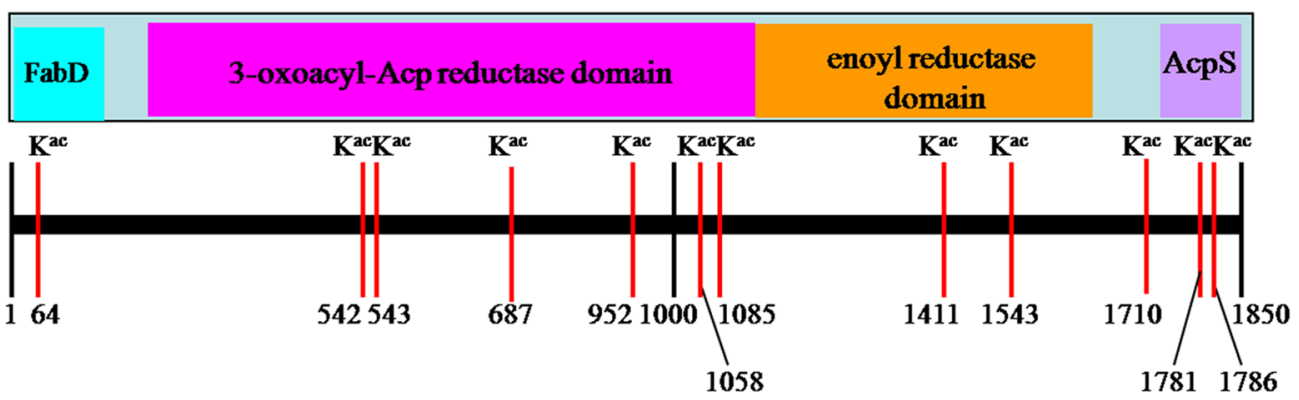

Fig. 8 Working scheme of lysine acetylated enzymes involved in central and triacylglycerols (TAGs) metabolism pathways in oleaginous yeasts. a Reconstruction of central and TAGs metabolism scheme from the KEGG pathway. Identified acetylated enzymes were highlighted in red. Abbreviations for the components, enzyme symbols and annotations are included in Additional file 8: Tables S7. b An overview of acetylation sites in fatty acid synthetase (FAS1). c An overview of acetylation sites in fatty acid synthetase (FAS2) 


\section{Acetylated proteins related to NADPH regeneration}

NADPH generated mainly by the malic enzyme (ME) (Ratledge and Wynn 2002), is indispensable for fatty acid biosynthesis. However, another research revealed that ME was not the key enzyme for fatty acid synthesis in $Y$. lipolytica, indicating that NADPH may derive from PPP pathway and generate via a cytosolic isocitrate dehydrogenase (ICDH) $\left(\mathrm{NADP}^{+}\right.$-dependent) (Dulermo et al. 2015). The results showed that two key enzymes in PPP pathway, glucose-6-phosphate dehydrogenase (GPDH) and 6-phosphogluconate dehydrogenase (PGD), which are responsible for generating NADPH, were both modified by acetyl groups (Fig. 8a). ICDH3, an isocitrate dehydrogenase $\left(\mathrm{NADP}^{+}\right.$-dependent), was also found to be acetylated (Additional file 8: Table S7). Besides GPDH, PGD and ICDH3, five proteins (accession no. Q6C1K2, Q6C2T9, Q6C351, Q6CBG4 and Q6CEH4) related to $\mathrm{NADPH}$ regeneration were also identified to be acetylated (Additional file 4: Table S3), reflecting a crucial role of this post-translational modification in NADPH regeneration.

Acetylated proteins involved in oxidative phosphorylation As ATP is one of the essential materials for fatty acid biosynthesis, we further investigated oxidative phosphorylation, a major biosynthesis process of ATP in Y. lipolytica.
The results showed that four electron- transferred complexs, including $\mathrm{NADH}$-coenzyme $\mathrm{Q}$ oxidoreductase (complex I), succinate-Q oxidoreductase (complex II), Q-cytochrome c oxidoreductase (complex III) and cytochrome c oxidase (complex IV), and ATP synthases were involved in acetylated (Fig. 9).

\section{Protein interaction network of acetylated proteins}

To investigate the cellular processes regulated by acetylation in Y. lipolytica, we used Cytoscape software to establish the protein-protein interaction network (Fig. 10). A total of 778 acetylated proteins were mapped to the protein interaction database (Additional file 9: Table S8), which presents a global view of how acetylated proteins perform various types of functions in $Y$. lipolytica. According to the algorithm of Cytoscape software, 26 highly interconnected clusters of acetylated proteins were retrieved (Additional file 9: Table S8). The top five clusters (Cluster I-V) identified were shown in Additional file 10: Figure S2, including ribosome, aminoacyl-tRNA biosynthesis, RNA transport, ribosome biogenesis in eukaryotes and oxidative phosphorylation. The complicated interaction networks of acetylated proteins indicate that the physiological interactions among these protein complexes are likely to contribute to their cooperation and coordination in the oleaginous yeast, $Y$. lipolytica.

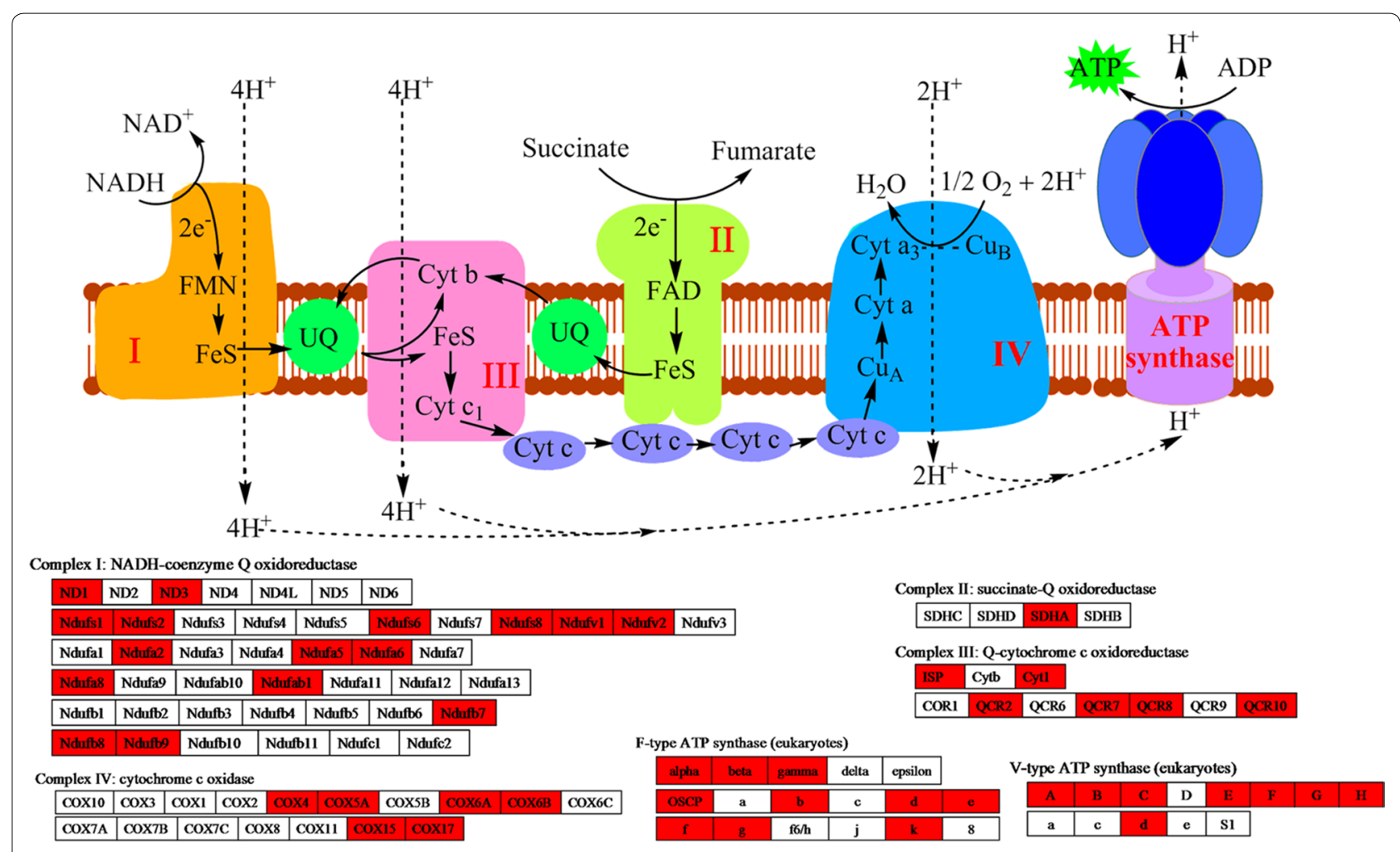

Fig. 9 Working scheme of lysine acetylation events involved in oxidative phosphorylation. Identified acetylated proteins were highlighted in red 


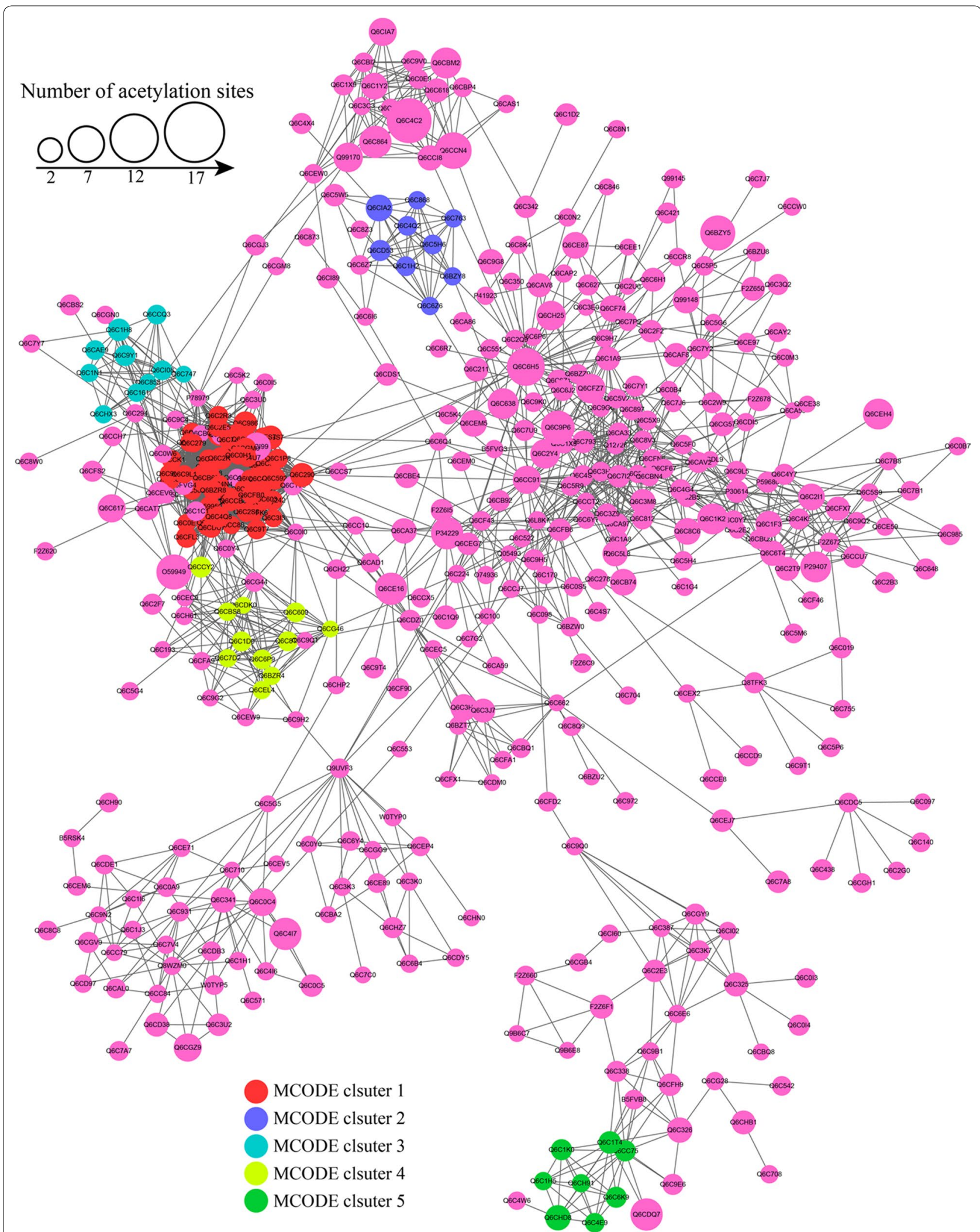

Fig. 10 Interaction networks of the acetylated proteins in oleaginous yeast. Interaction networks of all acetylated proteins with the top five clusters of proteins associated with ribosome (red), aminoacyl-tRNA biosynthesis (blue), RNA transport (light blue), ribosome biogenesis in eukaryotes (light green) and oxidative phosphorylation (green) 


\section{Discussion}

Lysine acetylation is a widespread and highly conserved post-translational modification in both eukaryotes and prokaryotes with multiple functions (Choudhary et al. 2009; Wang et al. 2010). Although many oleaginous microorganisms have been intensively investigated to produce microbial oils (Papanikolaou and Aggelis 2011; Ratledge and Wynn 2002), the regulatory mechanism of lipid biosynthesis in these organisms, especially the role of lysine acetylation in the regulation of these processes, is largely unknown. In the present study, we performed a proteomics study of lysine acetylation in the oleaginous yeast Y. lipolytica ACA-DC 50109.

A total of 1428 acetylated proteins with 3163 unique modification sites were identified, which is dramatically higher than those in other previously reported microorganisms (Table 1). The quantity of acetylated proteins was 1.35 times higher than that (1059) in S. cerevisiae (Henriksen et al. 2012), reflecting a potentially crucial role of lysine acetylation in Y. lipolytica. The acetylated proteins were distributed in multiple cellular compartments and participated in diverse functional biological processes, especially in lipid biosynthesis. Triacylglycerols (TAGs) is a key component of lipid droplets in oleaginous yeast (Papanikolaou and Aggelis 2011; Ratledge and Wynn 2002), which can be transformed into biodiesel by chemical ways or biological ways (Helwani et al. 2009). A large number of enzymes associated with TAGs biosynthesis were found to be acetylated (Fig. 8a), supporting the notion that lipid biosynthesis might be strictly regulated by lysine acetylation. Interestingly, two multi-enzymatic complexes of fatty acid synthetases, FAS1 and FAS2, contain 12 acetylation sites, respectively, which distribute in all the conserved domains of fatty acid synthetases.

NADPH and ATP are indispensable for fatty acid biosynthesis (Papanikolaou and Aggelis 2011; Ratledge and Wynn 2002). In this study, eight proteins related to NADPH regeneration were found to be modified by acetyl groups (Additional file 4: Table S3), reflecting a crucial role of this post-translational modification in NADPH regeneration. Several components important for ATP synthesis, including four electron-transferred complexs and two types of ATP synthases, were identified as acetylated proteins (Fig. 9). In support of our observations, some of the electron transfer related proteins, such as ferredoxin (FD) and ferredoxin $\mathrm{NADP}^{+}$reductase (FNR), were found to be lysine acetylated in cyanobacterium Synechocystis sp. (Mo et al. 2015). In addition, lysine acetylation was also observed on the b-subunit of chloroplastic ATP synthase in Arabidopsis (Wu et al. 2011) and rice (Xiong et al. 2016). All these findings highlight the notion that lysine acetylation might play a key regulatory role in the energy metabolic process.

To summarize, our results provide the first extensive data on lysine acetylation in oleaginous yeast $Y$. lipolytica. These data showed that lysine acetylation plays a critical regulatory role in diverse aspects of cellular process, especially in lipid biosynthesis. The dataset represents the first comprehensive view of the acetylome in oleaginous yeast and probably illuminates the crucial role of reversible acetylation in lipid biosynthesis and accumulation in oleaginous microorganisms.

Table 1 Comparison of Y. lipolytica acetylome with those of other published microorganisms

\begin{tabular}{|c|c|c|c|c|c|c|}
\hline Species & & No. of ORFs & No. of $\mathrm{K}^{\mathrm{ac}}$ sites & No. of $\mathrm{K}^{\mathrm{ac}}$ proteins & $\%$ of acetylation & References \\
\hline \multirow[t]{2}{*}{ Yeasts } & Y. lipolytica & 6472 & 3163 & 1428 & 22.1 & This study \\
\hline & S. cerevisiae & 5404 & 2878 & 1059 & 19.6 & Henriksen et al. (2012) \\
\hline \multirow[t]{3}{*}{ Fungi } & P. sojae & 19,027 & 2197 & 1150 & 6.0 & Li et al. (2016) \\
\hline & B. cinerea & 16389 & 1582 & 954 & 5.82 & Lv et al. (2016) \\
\hline & F. graminearum & 13,334 & 577 & 364 & 2.73 & Zhou et al. (2016) \\
\hline \multirow[t]{13}{*}{ Bacteria } & B. amyloliquefaciens & 3811 & 3268 & 1254 & 32.9 & Liu et al. (2016) \\
\hline & M. tuberculosis & 4034 & 1128 & 658 & 16.3 & Xie et al. (2015) \\
\hline & Synechocystis sp. & 3672 & 776 & 513 & 14.0 & Mo et al. (2015) \\
\hline & V.parahemolyticus & 4823 & 1413 & 656 & 13.6 & Pan et al. (2014) \\
\hline & S. reseosporus & 6891 & 1143 & 667 & 9.7 & Liao et al. (2014) \\
\hline & E. coli & 4144 & 1070 & 349 & 8.4 & Zhang et al. (2013) \\
\hline & T. thermophilus & 2238 & 197 & 128 & 5.7 & Okanishi et al. (2013) \\
\hline & P. aeruginosa & 5892 & 430 & 320 & 5.4 & Ouidir et al. (2015) \\
\hline & S. erythraea & 7198 & 664 & 363 & 5.0 & Huang et al. (2015) \\
\hline & B. subtilis & 4105 & 332 & 185 & 4.8 & Kim et al. (2013) \\
\hline & S. enterica & 4430 & 253 & 191 & 4.3 & Wang et al. (2010) \\
\hline & G. kaustophilus & 3532 & 253 & 114 & 3.2 & Lee et al. (2013) \\
\hline & E. amylovora & 3565 & 142 & 96 & 2.7 & Wu et al. (2013) \\
\hline
\end{tabular}




\section{Additional files}

Additional file 1: Figure S1. Representative MS/MS spectra of acetylpeptides from three proteins. (a) Acetylpeptide NLLTNFHGFDFTSDK(ac) $L R$ with an acetylation site at $K 109$ of the $40 S$ ribosomal protein S1 (Q6C2R9). (b) Acetylpeptide DK(ac)FDAAGIWYEHR with an acetylation site at $\mathrm{K} 246$ of the isocitrate dehydrogenase (Q6C2Y4). (c) Acetylpeptide DYFGAHTYQLLDGDGK(ac)WIHTNWTGR with an acetylation site at K469 of the 6-phosphogluconate dehydrogenase (Q6CEH4).

Additional file 2: Table S1. The identified acetylated sites in Y. lipolytica.

Additional file 3: Table S2. Protein GO enrichment based on molecular functions.

Additional file 4: Table S3. Protein GO enrichment based on biological process.

Additional file 5: Table S4. Protein GO enrichment based on cellular component.

Additional file 6: Table S5. Protein pathway enrichment.

Additional file 7: Table S6. Protein_domain_enrichment analysis.

Additional file 8: Table S7. Lysine acetylated enzymes related to central and lipid metabolisms in Y. lipolytica.

Additional file 9: Table S8. Protein interaction network of acetylated proteins.

Additional file 10: Figure S2. Interaction network of acetylated proteins associated with ribosome, aminoacyl-tRNA biosynthesis, RNA transport, ribosome biogenesis, and oxidative phosphorylation.

\section{Abbreviations}

AGC: automatic gain control; DTT: dithiothreitol; ECL: enhanced chemiluminescence; ESI/NSI: electrospray/nanospray ionization; FA: formic acid; FAS: fatty acid synthetase; FD: ferredoxin; FDR: false discovery rate; FNR: ferredoxin $\mathrm{NADP}^{+}$reductase; GO: gene ontology; GPDH: glucose-6-phosphate dehydrogenase; HATs: histone acetyltransferases; HDACs: histone deacetylases; HRP: horseradish peroxidase; IAA: iodoacetamide; KEGG: kyoto encyclopedia of genes and genomes; LC-MS/MS: liquid chromatography-mass spectrometry; ME: malic enzyme; PGD: 6-phosphogluconate dehydrogenase; PPP: pentose phosphate pathway; PTM: post-translational modification; PVDF: polyvinylidene difluoride; SDS-PAGE: sodium dodecyl sulfate polyacrylamide gel electropheresis; TAGs: triacylglycerols; TCA: tricarboxylic acid; YPD: yeast peptone dextrose.

\section{Authors' contributions}

GW, LG and LL designed research; GW, LG and LL performed research; WL contributed new reagents or analytic tools; $L L, G W, L G, Z C$ and $W L$ analyzed data; LL, GW, LG, ZC and WL wrote the paper. All authors read and approved the final manuscript.

\section{Author details}

${ }^{1}$ College of Life Sciences, Shandong Province Key Laboratory of Applied Mycology, Qingdao Agricultural University, Qingdao 266109, China. ${ }^{2}$ The Key Laboratory of Integrated Crop Pest Management of Shandong Province, College of Agronomy and Plant Protection, Qingdao Agricultural University, Qingdao 266109, China. ${ }^{3}$ College of Marine Life Sciences, Ocean University of China, Qingdao 266100, China.

\section{Acknowledgements}

This work was supported by the National Natural Science Foundation of China (31370779, 31401027), the Taishan Scholar Construction Foundation of Shandong Province (6631114314), the Project of Shandong Province Higher Educational Science and Technology Program (J15LE15, J16LE04), the Open Fund of Laboratory for Marine Biology and Biotechnology, Qingdao National Laboratory for Marine Science and Technology, Qingdao, China (No. OF2015NO19) and Research Foundation for Advanced Talents of Qingdao Agricultural University (6631114335).

\section{Competing interests}

The authors declare that they have no competing interests.

\section{Availability of data and materials}

The mass spectrometry proteomics data have been deposited to the Proteome $X$ change Consortium via the PRIDE partner repository.

\section{Funding}

This work was supported by the National Natural Science Foundation of China (31370779, 31401027), the Taishan Scholar Construction Foundation of Shandong Province (6631114314), the Project of Shandong Province Higher Educational Science and Technology Program (J15LE15, J16LE04), the Open Fund of Laboratory for Marine Biology and Biotechnology, Qingdao National Laboratory for Marine Science and Technology, Qingdao, China (No. OF2015NO19) and Research Foundation for Advanced Talents of Qingdao Agricultural University (6631114335).

\section{Publisher's Note}

Springer Nature remains neutral with regard to jurisdictional claims in published maps and institutional affiliations.

Received: 23 January 2017 Accepted: 26 April 2017

Published online: 12 May 2017

\section{References}

Allfrey VG, Faulkner R, Mirsky AE (1964) Acetylation and methylation of histones and their possible role in the regulation of RNA synthesis. Proc Natl Acad Sci USA 51:786-794

Chou MF, Schwartz D (2011) Biological sequence motif discovery using motifx. Curr Protoc Bioinform Chap 13:15-24

Choudhary C, Kumar C, Gnad F, Nielsen ML, Rehman M, Walther TC, Olsen JV, Mann M (2009) Lysine acetylation targets protein complexes and coregulates major cellular functions. Science 325(5942):834-840

Cox J, Mann M (2008) MaxQuant enables high peptide identification rates, individualized p.p.b.-range mass accuracies and proteome-wide protein quantification. Nat Biotechnol 26:1367-1372

Cox J, Matic I, Hilger M, Nagaraj N, Selbach M, Olsen JV, Mann M (2009) A practical guide to the MaxQuant computational platform for SILAC-based quantitative proteomics. Nat Protoc 4(5):698-705

Dulermo T, Lazar Z, Dulermo R, Rakicka M, Haddouche R, Nicaud JM (2015) Analysis of ATP-citrate lyase and malic enzyme mutants of Yarrowia lipolytica points out the importance of mannitol metabolism in fatty acid synthesis. Biochim Biophys Acta 1851:1107-1117

Folch J, Lees M. Sloane Stanley GH (1957) A simple method for the isolation and purification of total lipides from animal tissues. J Biol Chem 226:497-509

Helwani Z, Othman MR, Aziz N, Fernando WJN, Kim J (2009) Technologies for production of biodiesel focusing on green catalytic techniques: a review. Fuel Proc Technol 90:1502-1514

Henriksen P, Wagner SA, Weinert BT, Sharma S, Bacinskaja G, Rehman M, Juffer AH, Walther TC, Lisby M, Choudhary C (2012) Proteome-wide analysis of lysine acetylation suggests its broad regulatory scope in Saccharomyces cerevisiae. Mol Cell Proteom 11(11):1510-1522

Horton P, Park KJ, Obayashi T, Fujita N, Harada H, Adams-Collier CJ, Nakai K (2007) WoLF PSORT: protein localization predictor. Nucleic Acids Res 35(Web Server issue):W585-W587

Huang D, Li ZH, You D, Zhou Y, Ye BC (2015) Lysine acetylproteome analysis suggests its roles in primary and secondary metabolism in Saccharopolyspora erythraea. Appl Microbiol Biotechnol 99:1399-1413

Hunter S, Apweiler R, Attwood TK, Bairoch A, Bateman A, Binns D, Bork P, Das U, Daugherty L, Duquenne L, Finn RD, Gough J, Haft D, Hulo N, Kahn D, Kelly E, Laugraud A, Letunic I, Lonsdale D, Lopez R, Madera M, Maslen J, McAnulla C, McDowall J, Mistry J, Mitchell A, Mulder N, Natale D, Orengo C, Quinn AF, Selengut JD, Sigrist CJ, Thimma M, Thomas PD, Valentin F, Wilson D, Wu CH, Yeats C (2009) InterPro: the integrative protein signature database. Nucleic Acids Res 37(Database issue):D211-D215 
Jiao X, Sherman BT, da Huang W, Stephens R, Baseler MW, Lane HC, Lempicki RA (2012) DAVID-WS: a stateful web service to facilitate gene/protein list analysis. Bioinformatics 28(13):1805-1806

Kanehisa M, Goto S, Kawashima S, Okuno Y, Hattori M (2004) The KEGG resource for deciphering the genome. Nucleic Acids Res 32:D277-D280

Kim D, Yu BJ, Kim JA, Lee YJ, Choi SG, Kang S, Pan JG (2013) The acetylproteome of gram-positive model bacterium Bacillus subtilis. Proteomics 13(10-11):1726-1736

Lee KK, Workman JL (2007) Histone acetyltransferase complexes: one size doesn't fit all. Nat Rev Mol Cell Biol 8:284-295

Lee DW, Kim D, Lee YJ, Kim JA, Choi JY, Kang S, Pan JG (2013) Proteomic analysis of acetylation in thermophilic Geobacillus kaustophilus. Proteomics 13(15):2278-2282

Li D, Lv B, Tan L, Yang Q, Liang W (2016) Acetylome analysis reveals the involvement of lysine acetylation in diverse biological processes in Phytophthora sojae. Sci Rep 6:29897

Liao G, Xie L, Li X, Cheng Z, Xie J (2014) Unexpected extensive lysine acetylation in the trump-card antibiotic producer Streptomyces roseosporus revealed by proteome-wide profiling. J Proteom 106:260-269

Liu L, Wang G, Song L, Lv B, Liang W (2016) Acetylome analysis reveals the involvement of lysine acetylation in biosynthesis of antibiotics in Bacillus amyloliquefaciens. Sci Rep 6:20108

Lv B, Yang Q, Li D, Liang W, Song L (2016) Proteome-wide analysis of lysine acetylation in the plant pathogen Botrytis cinerea. Sci Rep 6:29313

Mischerikow N, Heck AJ (2011) Targeted large-scale analysis of protein acetylation. Proteomics 11:571-589

Mo R, Yang M, Chen Z, Cheng Z, Yi X, Li C, He C, Xiong Q, Chen H, Wang Q, Ge $\mathrm{F}$ (2015) Acetylome analysis reveals the involvement of lysine acetylation in photosynthesis and carbon metabolism in the model cyanobacterium Synechocystis sp. PCC 6803. J Proteome Res 14(2):1275-1286

Nambi S, Gupta K, Bhattacharyya M, Ramakrishnan P, Ravikumar V, Siddiqui N, Thomas AT, Visweswariah SS (2013) Cyclic AMP-dependent protein lysine acylation in mycobacteria regulates fatty acid and propionate metabolism. J Biol Chem 288(20):14114-14124

Okanishi H, Kim K, Masui R, Kuramitsu S (2013) Acetylome with structural mapping reveals the significance of lysine acetylation in Thermus thermophilus. J Proteome Res 12:3952-3968

Ouidir T, Cosette P, Jouenne T, Hardouin J (2015) Proteomic profiling of lysine acetylation in Pseudomonas aeruginosa reveals the diversity of acetylated proteins. Proteomics 15:2152-2157

Pan J, Ye Z, Cheng Z, Peng X, Wen L, Zhao F (2014) Systematic analysis of the lysine acetylome in Vibrio parahemolyticus. J Proteome Res 13:3294-3302

Papanikolaou S, Aggelis G (2003) Selective uptake of fatty acids by the yeast Yarrowia lipolytica. Eur J Lipid Sci Technol 105:651-655

Papanikolaou S, Aggelis G (2011) Lipids of oleaginous yeasts. Part I: biochemistry of single cell oil production. Eur J Lipid Sci Technol 113:1031-1051

Phillips DM (1963) The presence of acetyl groups of histones. Biochem J 87:258-263
Ratledge C, Wynn JP (2002) The biochemistry and molecular biology of lipid accumulation in oleaginous microorganisms. Adv Appl Microbiol 51:1-51

Santos AP, Serra T, Figueiredo DD, Barros P, Lourenco T, Chander S, Oliveira MM, Saibo NJ (2011) Transcription regulation of abiotic stress responses in rice: a combined action of transcription factors and epigenetic mechanisms. OMICS 15(12):839-857

Shannon P, Markiel A, Ozier O, Baliga NS, Wang JT, Ramage D, Amin N, Schwikowski B, Ideker T (2003) Cytoscape: a software environment for integrated models of biomolecular interaction networks. Genome Res 13(11):2498-24504

Spange S, Wagner T, Heinzel T, Kramer OH (2009) Acetylation of non-histone proteins modulates cellular signalling at multiple levels. Int J Biochem Cell Biol 41:185-198

Starai VJ, Escalante-Semerena JC (2004) Identification of the protein acetyltransferase (Pat) enzyme that acetylates acetyl-CoA synthetase in Salmonella enterica. J Mol Biol 340:1005-1012

Wang $Q$, Zhang Y, Yang C, Xiong H, Lin Y, Yao J, Li H, Xie L, Zhao W, Yao Y, Ning ZB, Zeng R, Xiong Y, Guan KL, Zhao S, Zhao GP (2010) Acetylation of metabolic enzymes coordinates carbon source utilization and metabolic flux. Science 327(5968):1004-1007

Wang GY, Chi Z, Song B, Wang ZP, Chi ZM (2012) High level lipid production by a novel inulinase-producing yeast Pichia guilliermondii Pcla22. Bioresour Technol 124:77-82

Wang ZP, Xu HM, Wang GY, Chi Z, Chi ZM (2013) Disruption of the MIG1 gene enhances lipid biosynthesis in the oleaginous yeast Yarrowia lipolytica ACA-DC 50109. Biochim Biophys Acta 1831:675-682

Wang GY, Zhang Y, Chi Z, Liu GL, Wang ZP, Chi ZM (2015) Role of pyruvate carboxylase in accumulation of intracellular lipid of the oleaginous yeast Yarrowia lipolytica ACA-DC 50109. Appl Microbiol Biotechnol 99:1637-1645

Wu X, Oh MH, Schwarz EM, Larue CT, Sivaguru M, Imai BS, Yau PM, Ort DR, Huber SC (2011) Lysine acetylation is a widespread protein modification for diverse proteins in Arabidopsis. Plant Physiol 155(4):1769-1778

Wu X, Vellaichamy A, Wang D, Zamdborg L, Kelleher NL, Huber SC, Zhao Y (2013) Differential lysine acetylation profiles of Erwinia amylovora strains revealed by proteomics. J Proteom 79:60-71

Xie L, Wang X, Zeng J, Zhou M, Duan X, Li Q, Zhang Z, Luo H, Pang L, Li W, Liao G, Yu X, Li Y, Huang H, Xie J (2015) Proteome-wide lysine acetylation profiling of the human pathogen Mycobacterium tuberculosis. Int J Biochem Cell Biol 59:193-202

Xiong Y, Peng X, Cheng Z, Liu W, Wang GL (2016) A comprehensive catalog of the lysine-acetylation targets in rice (Oryza sativa) based on proteomic analyses. J Proteom 138:20-29

Yang XJ, Seto E (2008) Lysine acetylation: codified crosstalk with other posttranslational modifications. Mol Cell 31:449-461

Zhang K, Zheng S, Yang JS, Chen Y, Cheng Z (2013) Comprehensive profiling of protein Iysine acetylation in Escherichia coli. J Proteome Res 12:844-851

Zhou S, Yang Q, Yin C, Liu L, Liang W (2016) Systematic analysis of the lysine acetylome in Fusarium graminearum. BMC Genom 17:1019

\section{Submit your manuscript to a SpringerOpen ${ }^{\odot}$ journal and benefit from:}

- Convenient online submission

- Rigorous peer review

- Immediate publication on acceptance

- Open access: articles freely available online

- High visibility within the field

- Retaining the copyright to your article

Submit your next manuscript at springeropen.com 